\title{
Vortex Motions in Ideal Bose Superfluid*
}

\author{
Martin J. Cooper \\ Institute for Basic Standards, National Bureau of Standards, Washington, D.C. 20234 \\ (February 11, 1969)

\begin{abstract}
A general nonlinear field equation is derived for the macroscopic order parameter of an ideal coherent Bose gas. It is shown that this noninteracting system can support stable quantized vortexlike motions within the superfluid phase. It is suggested that this coherent phase of the ideal Bose gas describes the dominant physical features of real superfluid liquid helium.
\end{abstract}

Key words: Coherent Bose gas; superfluid; vortices.

\section{Introduction}

The presence of quantized vortex motions in the superfluid phase of liquid helium was first postulated by Onsager [1] ${ }^{1}$ and Feynman [2] and has since been confirmed by numerous experiments [3]. The microscopic theory of such macroscopic motions was developed independently by Gross [4] and Pitaevskii [5], who assumed the fluid to be a dilute gas of weakly repulsive Bose particles. They were able to show that a self-consistent equation-of-motion for the macroscopic order parameter of the Boson field contains a nonlinear contribution arising from the interactions between the particles. Stable vortex motions with quantized circulation appear as special solutions of the nonlinear field equation.

In this paper we suggest that vortex motions of the superfluid phase are due to the Bose statistics and are not dependent upon any interactions (either attractive or repulsive) between the particles. We shall demonstrate this by showing that quantized vorticles are stable configurations of the macroscopic order parameter within the ordered phase of a noninteracting free Bose gas.

It is generally remarked that the ideal Bose gas is an overly simple system which describes unphysical situations due to its oversensitivity to boundary conditions. If, for example, the gas is contained in a rigid box of large but finite dimensions, it is found that the particles will condense into a ground state with a highly nonuniform density profile [4]. Such an unreal distribution is usually removed by introducing repulsive interactions between the particles so that they smear themselves out more uniformly over the entire volume. Since we wish to exhibit

\footnotetext{
*Not subject to copyright-contribution of the National Bureau of Standards.

Figures in brackets indicate the literature references at the end of this paper.
}

certain properties as explicit characteristics of the Bose statistics in the absence of any interactions and independent of boundary conditions, we choose instead to think of the ideal Bose system within the framework of the Grand Canonical formalism. This permits us to consider an ensemble of $N$ noninteracting Bosons contained in a volume $V\left(=L^{3}\right)$ at an equilibrium temperature $T(=1 / \beta)$ in the thermodynamic limit of $N \rightarrow \infty, V \rightarrow \infty$ with a fixed number density $N / V$.

\section{Field Equation}

The appropriate description for the ideal gas of Boson particles is given in terms of the creation and annihilation operators of the quantized Bose field, $\psi^{+}(r), \psi(r)$ with $\left[\psi(r), \psi^{+}\left(r^{\prime}\right)\right]=\delta\left(r-r^{\prime}\right)$. The presence of an ordered or condensed phase is characterized by the existence of a spontaneous macroscopic expectation value for the single operator $\psi(r)[6]$. This author together with Green [7] have considered the condensation of such a system as a model secondorder phase transition by removing the phase symmetry of the Fock states through a linear coupling of the field operators to a fictitious external source $S(r)$. Making use of the coherent state representation and following standard thermodynamic arguments, it was shown that the number density is of a simple analytic form. In the limit of a vanishing uniform source $S(r)=S \rightarrow 0$ (after the thermodynamic limit of $N, V \rightarrow \infty, N / V$ finite) the number density separates into two branches at a critical value of the temperature, $T_{c}$. Below this temperature, the total density is composed of two uniform coexisting parts,

$$
n=\eta T^{3 / 2}+\Psi^{2}
$$


where the constant $\eta=(2 \pi \hbar / m)^{3 / 2} \zeta\left(\frac{3}{2}\right)=(2 \pi)^{3 / 2}$ 2.612 . . in units of $\hbar=m=1$. The first term is just the number density for the normal component of the fluid. The quantity $\Psi$ corresponds to a spontaneous thermal expectation value which is of finite magnitude below $T_{c}$ and vanishes identically for all temperatures above it. In general, $\Psi$ represents a complex order parameter for the coherent phase with both an amplitude and phase $\Psi=|\Psi(r)| \exp (i \phi(r))$. Within the framework of the two fluid model, $|\Psi|^{2}$ is associated with the number density of the ordered or superfluid phase, while the spatial gradient of the phase is interpreted as the superfluid velocity $v_{s}=\nabla \phi(r)$.

The thermodynamic potential describing the coherent phase as a function of the superfluid density $|\Psi|^{2}$ and temperature $T$ in the vicinity of the transition as $\left(T-T_{c}\right) / T_{c}=t<0$ becomes small is given by

$$
F\left(\Psi^{2}, T\right)=\left[\Psi^{2}\left(1+\frac{3}{2} t / \Psi^{2}\right)\right]^{3}
$$

Taking the actual superfluid to be locally a coherent Bose gas, $[7,8]$ this result provides the functional form of the thermodynamic potential density expressed in terms of the reduced temperature $t$ and the magnitude of local macroscopic order parameter $\Psi(r)$. It is like a phenomenological Landau expansion for the free-energy carried to third order in $\Psi^{2}$. If the superfluid is spatially nonuniform or in motion relative to the normal component $\left(v_{n}=0\right)$ then there is an additional contribution $\hbar^{2}|\nabla \Psi|^{2} / 2 m[9]$.

[Remark-We wish to note here the similarity between the thermodynamic potential for the coherent superfluid phase of the ideal Bose fluid given in eq (2) and certain recently proposed forms for the potential of superfluid helium. Several persons [10-12] have suggested variations of the conventional Landau theory of phase-transitions based on the expansion of $F\left(\Psi^{2}, T\right)$. Noting that the superfluid density varies like $\left(1-T / T_{\lambda}\right)^{2 / 3}$, Mamaladze[10] made ad hoc modifications in the temperature dependence of the lowest order expansion coefficients. Amit[11] proposed a more consistent formulation by including terms to order $\Psi^{6}$ and has been able to describe certain features of superfluid helium films. Miller and Luban[12] pointed out a further consequence of such a term would be the existence of two critical superfluid velocities.]

For stable configurations, the total potential function [9] $\mathscr{F}\left(\Psi^{2}, \quad T\right)=\int d r\left[-\frac{1}{2}|\nabla \Psi|^{2}+F\left(\Psi^{2}, \quad T\right)\right]$ must be a minimum with respect to $\Psi$ (also, its conjugate $\left.\Psi^{*}\right)$. The variational procedure leads to a field equation for the magnitude of the local order parameter $\Psi(r)$ which describes the superfluid behavior of the coherent ideal Bose gas,

$$
\nabla^{2}|\Psi(r)|+\left(\Psi^{2}+\frac{3}{2} t\right)^{2}|\Psi(r)|=0 .
$$

This result is like the equation obtained by Gross[4] and Pitaevskii[5] to describe the superfluid properties of an interacting Bose gas. Taking a finite fraction of the particles to be in a single quantum state, they showed that the condensate wave function $\psi$ behaves like an effective hydrodynamic variable which obeys a nonlinear equation

$$
\nabla^{2} \psi(r)+\left(1-2 V \psi^{2}\right) \psi=0 .
$$

The theory is restricted by the requirement that the product of the condensate density and the effective potential, $|\psi|^{2} V$ remain small. A basic potential dependent dimension $\lambda=\left(2|\psi|^{2} V\right)^{-1 / 2}$ is used to characterize the length scale. The nonlinear contribution to the equation is directly proportional to the interaction potential. Thus, in the absence of such interactions, the $G-P$ equation describes a simple free-field whose characteristic length becomes infinite.

The field describing the hydrodynamics of the superfluid phase of the coherent Bose gas is given by eq (3). There, despite the lack of any direct potential, the equation contains terms to order $|\Psi|^{5}$ and is therefore of a more nonlinear nature than that described by the $G-P$ result.

\section{Special Solutions}

We shall consider solutions of the field equation for $\Psi$ in two cases of particular physical interest, namely, the behavior of the superfluid near a wall and the possibility of vortex-like configurations. It is convenient to obtain a normalized field equation by introducing a reduced variable for the magnitude of the order parameter

$$
\psi=\left(\frac{2}{3}|t|\right) 1 / 2|\Psi(r)|
$$

and a new distance scale $\xi=\left(\frac{27}{2}\right)^{1 / 2}|t| r$

$$
\nabla^{2} \psi+\left(1-\psi^{2}\right)^{2} \psi=0
$$

Consider first the problem of the Bose gas in a semiinfinite domain bounded by rigid wall ( $x-y$ plane). In this one dimensional geometry, the superfluid density vanishes at the wall $\left(\psi^{2}=0, z=0\right)$ and has a uniform value (normalized $\psi^{2}=1$ ) far from it. With these boundary conditions, the solution of eq. (5) is given by

$$
\psi(z)=z /\left(3+z^{2}\right)^{1 / 2} .
$$

This implies that the superfluid density $\psi^{2}$ rises from zero to over half of its uniform value less than two distance units from the wall $(3 / 4$ of its uniform value by three distance units). It appears therefore that the coherent superfluid phase of the ideal Bose gas is strongly "self-healing" and not overly sensitive boundary conditions.

The occurrence of a natural length scale parameter as a general consequence of the nonlinear equation for the interacting Bose gas has already been noted. In the same one dimensional geometry, the $G-P$ equation has the solution $\psi=(2 V)^{-1 / 2} \tanh (z / 2)$. 
Here, the corresponding healing behavior of the superfluid density depends inversely on the strength of potential. Thus, as the interactions between the particles becomes small, the healing scale becomes increasingly large. As the potential vanishes, the characteristic length scale $\lambda$ diverges, typifying the over response of the noninteracting Bose gas within the usual treatment.

The nonlinear nature of the field equation for the order parameter $\psi$ suggests that vortex-like motions may be stable configurations within the ideal Bose superfluid [13]. This can be demonstrated by noting that the field equation (eq (5)) permits cylindrically symmetric solutions of the form $\psi(r, \theta, z)=R(r) \exp (i \nu \theta)$, where $\nu$ is an integer. The radial function $R(r)$, which is normalized by requiring that $2 \pi \int d r r|R(r)|^{2}=$ superfluid density per unit height along the $z$ axis, satisfies the differential equation

$$
\left(\frac{1}{r} \frac{d}{d r} r \frac{d}{d r}-\frac{\nu^{2}}{r^{2}}\right) R+\left(1-R^{2}\right)^{2} R=0 .
$$

While an explicit solution of this equation has not in general been possible, the asymptotic properties of the radial function can be determined [14]. Near the axis as $r \rightarrow 0$, the centrifugal force term dominates so that $R(r)$ must vanish like $r^{+|\nu|}$. Within this region the higher powers of $R$ are negligible and the radial behavior may be approximated by the Bessel function of order $|\nu|, R(r) \sim J_{|\nu|}(r)$, (to within a constant multiplicative factor). At very large distances from the axis, the superfluid density approaches its uniform value, $\psi^{2}(r \rightarrow \infty)=1$, with the radial function of the asymptotic form $R(r) \simeq-|\nu| / r$. When $\nu=0$, only the simple solution of an everywhere uniform $R(r)=1$ is possible.

These cylindrical solutions for the quantum-mechanical order parameter $\psi$ describe a field with a macroscopic axial component of angular momentum $L_{z}$,

$$
L_{z} \equiv \frac{\hbar}{2 i} \int d r\left[\psi^{*} \frac{\partial \psi}{\partial \theta}-\frac{\partial \psi^{*}}{\partial \psi} \psi\right]=\nu \int d r|R(r)|^{2}
$$

Thus, the total angular momentum is proportional to the superfluid density, $\psi^{*} \psi$. The flow field of the superfluid about the $z$ axis has a tangential component of angular velocity $v_{\theta}$,

$$
v_{\theta} \equiv \frac{\hbar}{2 i}\left[\psi^{*} \frac{1}{r} \frac{\partial \psi}{\partial \theta}-\frac{1}{r} \frac{\partial \psi^{*}}{\partial \theta} \psi\right] / \psi^{*} \psi
$$

which falls off like $|\nu| / r$. Such a solenoidal flow pattern is characteristic of a "classical vortex line" found in the hydrodynamic theory of incompressible fluids. The vortex filament has a vorticity $\Omega_{z} \equiv(\operatorname{curl} v)_{z}$ which is zero everywhere (except on the axis where the superfluid density vanishes such that the strength $\left(\Omega_{z}|\psi|^{2}=0, r=0\right)$. The circulation of the vortex flow

$$
\Gamma=\oint d \theta r v_{\theta}=\hbar|\nu|>0
$$

is quantized in units of $\hbar$.
One may estimate the effective size of the vortex core by setting the two asymptotic forms of $R(r)$ equal at a common value $r=\rho$ [11]. Requiring also continuity of the radial derivatives,

$$
\begin{aligned}
& A J_{|\nu|}(r) \simeq 1-\nu / r \\
& A J_{|\nu|}^{\prime}(r) \simeq \nu / r^{2} \quad(r=\rho)
\end{aligned}
$$

we find a core dimension $\rho=(1+|\nu|) /|\nu|$.

Gross [4] and Pitaevskii [5] found similar vortex-like solutions to be stable configurations in the superfluid phase of the interacting Bose gas. In that system, the vortices owe their existence to the presence of the nonlinear term in the $\mathrm{G}-\mathrm{P}$ field equation arising from the repulsive interactions between the particles. There the asymptotic solutions of the $\mathrm{G}-\mathrm{P}$ radial equation are given by

$$
\begin{aligned}
& R(r \approx 0) \simeq J_{|\nu|}(r) \\
& R(r \gg 0) \simeq 1-|\nu|^{2} / 2 r^{2} .
\end{aligned}
$$

While these limiting forms for the radial behavior are independent of the interactions, the order parameter itself depends upon the strength of the potential through the normalization $\psi=(2 V)^{1 / 2} R(r) \exp (i|\nu| \theta)$. Thus in the absence of any interactions between particles, such vortex lines vanish as possible solutions to the $\mathrm{G}-\mathrm{P}$ field equation.

In general it is not possible to obtain explicit solutions to the field equation for the order parameter of either the ideal Bose system (eq (3)) or the interacting gas ( $\mathrm{G}-\mathrm{P}$ equation) [15]. Both are highly nonlinear equations and can be solved only for certain very special situations. In the limit of a low superfluid density to a first approximation, they are both nonlinear to the same order $\psi^{3}$, and will describe a somewhat similar type of physical behavior. It is, however, important to recognize the very essential difference in the physical origin of the nonlinearity. In the G-P equation, the nonlinear term arises from the direct repulsive interactions between the particles which are superimposed upon the Bose statistics. Without these interactions, the super-fluid order parameter satisfies a linear equation characteristic of a free noninteracting quantum field. Quite a different behavior was found for the superfluid phase of the ideal coherent Bose gas. In that description, since all terms in the field equation are due to the particle statistics, the nonlinear nature of the order parameter is a direct consequence of the Bose statistics. While any real gas must certainly have strong short-ranged repulsive interactions between particles, this nonlinear behavior suggests that the coherent phase of the ideal Bose gas might well describe the dominant physical features of superfluid liquid helium. 


\section{References}

[1] Onsager, L., Nuovo Cimento, S. 6, 249 (1949).

[2] Feynman, R., Progress in Low Temperature Physics (NorthHolland Publ. Co., Amsterdam, 1955) I, p. 17.

[3] Vinen, W., Proc. Roy. Soc. (London) A260, 218 (1961), Rayfield, G. W., and Reif, F., Phys. Rev. Letters 11, 305 (1963). Also, review article by Andronikoshville, E., and Mamaladze, Y., Rev. Mod. Phys. 38, 567 (1966).

[4] Gross, E. P., Nuovo Cimento 20, 454 (1961); J. Math. Phys. 4, 195 (1963).

[5] Pitaevskii, L. P., Sov. Phys. J.E.T.P. 40, 646 (1961).

[6] An excellent discussion of the macroscopic mean-value of a quantum-field operator as the appropriate thermodynamic order parameter for quantum fluids is given by Anderson, P., Rev. Mod. Phys. 38, 298 (1966).

[7] Cooper, M. J., and Green, M. S., Phys. Rev. 176, 302 (1968).

[8] The use of the coherent state representation to describe ideal Bose condensation and its further application to the theory of superfluids has been discussed by a number of persons. [Casher, A.. and Revzan, M., Amer. J. Phys. 35, 1154 (1968); Cummins, F. W., and Johnston, J. R., Phys. 151, 105 (1966); Langer, J. S., Phys. Rev. 167, 183 (1968).] Particularly rele- vant is the work of Langer in which a free-energy functional like that described here is obtained by the expansion of an exponential form containing a slowly varying $\psi(r)$.

[9] The complete expression for $F\left(\psi^{2}, T\right)$ appropriate to a nonuniform system is given in eq (34) of ref. 7.

[10] Mamaladze, Y. G., Sov. Phys. J.E.T.P. 25, 479 (1967).

[11] Amit, D., Phys. Letters 26A, 448 and 466 (1968).

[12] Luban, M., and Miller, S., Phys. Letters 27A, 501 (1968).

[13] The development in this section follows the argument presented by Gross in describing vortices in the interacting Bose condensate.

[14] The radial equation obtained from the $\mathrm{G}-\mathrm{P}$ equation for the interacting Bose gas is given by

$$
r^{2} R^{\prime \prime}+r R^{\prime}-\nu^{2} R+r^{2}\left(1-2 V R^{2}\right) R=0 .
$$

Explicit solution of this equation has not been possible over the entire range for $r$. The original work of Ginsburg, V., and Pitaevskii, L., contains a numerical solution, see Sov. Phys. J.E.T.P. 7, 858 (1958).

[15] Some special solutions of the $\mathrm{G}-\mathrm{P}$ equation appropriate to the description of physical problems are given by Wu, T. T., J. Math. Phys. 2, 105 (1961). See also references 4, 5.

(Paper 73A3-554) 